\section{Globale Großmachtrivalität}

Angela Stent: Russia and China. Axis of Revisionists?

Washington, D.C.: The Brookings Institution, February 2020

Besprochen von Dr. Hannes Adomeit: Senior Fellow, Institut für Sicherheitspolitik an der Universität Kiel (ISPK), E-Mail: Hannes.adomeit@t-online.de

https://doi.org/10.1515/sirius-2020-2020

Es ist nicht klar, warum ein Fragezeichen hinter dem Titel der Studie über die russisch-chinesischen Beziehungen als Achse zwischen zwei revisionistischen Mächten steht. Die Autorin, Angela Stent, Direktorin des Center for Eurasian, Russian and East European Studies der Georgetown University und Nonresident Senior Fellow an der Brookings Institution, schreibt, dass China und Russland revisionistische Mächte seien, weil sie „gemeinsam eine globale postWest Ordnung schaffen wollen, die ihre Interessen berücksichtigt und ihrer autoritären Herrschaft dienlich ist.“

Im Einzelnen argumentiert die Autorin, dass die Beziehungen zwischen China und Russland seit 2014 $\mathrm{zu}$ einer zunehmend robusten, pragmatischen strategischen Partnerschaft geworden seien. Seit der Besetzung und Annexion der Krim durch Russland im Jahr 2014 und dem anschließenden Bemühen des Westens, Russland zu isolieren, habe sich Putin zunehmend China zugewandt. Dies habe es dem Kreml ermöglicht, die internationale Isolation des Landes zu überwinden und erfolgreich auf der Weltbühne zu agieren. China sei Russlands größter
Handelspartner und der zweitgrößte Abnehmer russischer militärischer Hardware. Die Wirtschaftsbeziehungen nähmen zu. Die neue Gaspipeline Power of Siberia werde ihre gegenseitige Abhängigkeit von Energie noch erhöhen. Die chinesisch-russische Zusammenarbeit im Militärbereich vertiefe sich mit gemeinsamen Militärübungen und Luftpatrouillen, ebenso ihre Zusammenarbeit bei der Entwicklung künstlicher Intelligenz und Biotechnologie.

Die Darstellung des Verhältnisses in den Kapiteln „Vom chinesisch-sowjetischen Schisma bis zur Krise in der Ukraine“, „die Inneren Antriebe der Beziehungen“, „Wirtschaft und Energie, „Militärbeziehungen“, „High-Tech Zusammenarbeit“, und „die Politischen Beziehungen“ ist kurz, knapp und bündig, bringt aber wenig Neues. Von Interesse sind jedoch insbesondere wegen der guten Vernetzung der Autorin in der US-amerikanischen akademischen Community und dem Außenministerium und dem Einfluss, den sie auf die Diskussion und Gestaltung der Russland-Politik Washingtons ausübt, die Kapitel über die „Künftigen Herausforderungen“ und die „Implikationen für die amerikanische Politik“.

Mit den Herausforderungen sind die aktuellen und potenziellen Probleme gemeint, die sich aus den erheblichen Asymmetrien im Verhältnis der beiden Mächte ergeben. Die bestünden erstens in den Auswirkungen, die Chinas ehrgeizige „Gürtel und Straße“-Initiative auf die politischen und wirtschaftlichen Interessen Russlands haben könnten. Die Initiative werde den globalisierten Handel, die Finanzierungsströme verbreitern, Infrastrukturprojekte fördern und Märkte für chinesische Waren entwickeln. Die Transportkorridore, die China im Rahmen seiner Initiative schaffen will, würden jedoch Russland im Süden weitgehend umgehen. Daher sei es ungewiss, wie Russland von den massiven Infrastrukturprojekten profitieren soll. Sicher werde sich aber der geopolitische Einfluss Chinas erweitern. Es werde sich unweigerlich stärker für die Sicherheit seiner Investitionen in den Ländern einsetzen, durch die seine Autobahnen, Eisenbahnen und Pipelines verlaufen. Die bisherige „Arbeitsteilung“ zwischen Russland und China in Zentralasien könnte sich in einer Weise ändern, die den Interessen des Kremls zuwiderläuft.

Zweitens seien Russland und China keine natürlichen Verbündeten. Die meisten Russen identifizierten sich als Europäer und hätten kulturell viel mehr mit Europa und den Vereinigten Staaten gemeinsam als mit China. Wohin beispielsweise schickten die Chinesen ihre Kinder zum Lernen? In den Vereinigten Staaten gibt es mehr als 350.000 chinesische Studenten. Im Gegensatz dazu sind es in ganz Russland nur 25.000. Dies spiegle die breitere Wirklichkeit wider, dass die Kontakte der Zivilgesellschaft 
zwischen Russen und Chinesen weitaus geringer seien als die zwischen Russland und dem Westen oder China und dem Westen.

Drittens sei China wirtschaftlich und militärisch eine aufstrebende Macht, dessen Wirtschaft mehr als achtmal so groß wie diejenige Russlands sei. Moskau könne zwar militärische Macht effektiv auf der Weltbühne projizieren, bliebe jedoch ein Waffen- und Rohstoffexportland mit geringen Wachstumsraten und einer Wirtschaft, die sich nicht modernisiert und in Stagnation verharrt. Russland sei der Juniorpartner in dieser Beziehung, ein Status, den es offenbar akzeptiert habe, allerdings wohl nur solange wie es von China als gleichwertig behandelt und nicht direkt in seiner gemeinsamen Nachbarschaft herausgefordert werde.

Viertens seien chinesische und russische Visionen der „post-West“ globalen Ordnung, für die sie sich einsetzen, sehr unterschiedlich. Die Autorin zitiert in diesem Zusammenhang einen hochrangigen chinesischen Beamten mit den Worten: „China und Russland haben unterschiedliche Einstellungen. Russland will die derzeitige internationale Ordnung zerstören. Russland glaubt, es sei das Opfer des gegenwärtigen internationalen Systems, in dem sich seine Wirtschaft und seine Gesellschaft nicht entwickeln könne. China profitiert jedoch vom derzeitigen internationalen System. Wir wollen es verbessern und modifizieren, nicht zerstören." Stent greift dieses Argument zustimmend auf und argumentiert weiter, dass Russland eine multipolare Weltordnung anstrebe, in der die Vereinigten Staaten ihre Bedingungen nicht mehr diktieren könnten, ein ,dreigliedriges Jalta“, in dem Russland, China und die Vereinigten Staaten ihre Einflusssphären abgrenzten. Pekings Vision einer zukünftigen Weltordnung bestünde dagegen in den Großen Zwei, China und den Vereinigten Staaten, wobei Russland neben Europa, Japan und Indien seine sekundäre Rolle spielen würde.

Ungeachtet der Asymmetrien in den chinesisch-russischen Beziehungen hätten die beiden Länder jedoch zwingende Gründe, ihre Partnerschaft aufrecht zu erhalten und sogar zu stärken. Dafür spreche das ihnen gemeinsame Misstrauen gegenüber den Vereinigten Staaten und ihr Engagement für die Schaffung einer globalen Ordnung, die es ihnen ermögliche, ihre autoritären Regime aufrechtzuerhalten. Einige Analysten in den USA glaubten, es sei möglich, Russland von China ,abzulösen“ und dass Moskau bereit sein könnte, sich von China zu distanzieren und sich mit Washington gegen Peking neu zu positionieren. Dafür notwendig wäre, dass der US-Kongress die gegen Russland verhängten Sanktionen aufheben und Verhandlungen mit Russland zur Verlängerung des Neuen START-Rüstungskontrollabkommen aufnähme. Weitere und weitergehende Schritte, die die Vereinigten Staaten gehen könnten, wären, die Eingliederung der Krim in die Russische Föderation anzuerkennen und zu vereinbaren, dass die Ukraine ein neutrales Land bleiben und niemals der NATO beitreten würde. Allerdings kommt Stent zu dem (allerdings nicht so klar und eindeutig wie wünschenswert formulierten) Ergebnis, dass der Kongress schon nicht der Aufhebung von Sanktionen zustimmen würde. Und auch wenn Washington dennoch diese und weitere Bedingungen des Kremls zur nachhaltigen Verbesserung der Beziehungen annähme, würde sich Russland doch nicht von China distanzieren und die Dynamik der chinesisch-russischen militärischen, technologischen und wirtschaftlichen Zusammenarbeit umkehren.

https://www.brookings.edu/research/russia-andchina-axis-of-revisionists/ 